УДК 621.315 .592

\title{
Термоэлектрические интенсификаторы теплообмена
}

\author{
() И.А. Драбкин, Л.Б. Ершова \\ $\mathrm{OOO}$ „PMT“, \\ 115230 Москва, Россия \\ E-mail: igordrabk@gmail.com \\ Поступила в Редакцию 18 августа 2021 г. \\ В окончательной редакции 25 августа 2021 г. \\ Принята к публикации 25 августа 2021 г.
}

\begin{abstract}
Рассматривается работа термоэлектрического интенсификатора теплообмена. Приводятся критерии эффективности применения таких интенсификаторов и на этой основе анализируется их работа. Даются оценки положительного эффекта от применения термоэлектрического модуля в зависимости от разности температур на теплообменнике без использования термоэлектрического модуля. Показано, что в большинстве случаев использование интенсификатора не способствует интенсификации теплообмена.
\end{abstract}

Ключевые слова: термоэлектрический охладитель, теплообмен, интенсификатор, эффективность.

DOI: $10.21883 /$ FTP.2022.01.51802.04

\section{1. Введение}

Для понижения температур тепловыделяющих объектов иногда рекомендуют использовать термоэлектрический охладитель, за счет работы которого понижается температура объекта, а температура теплосбрасывающего устройства возрастает, что увеличивает сброс тепла в окружающую среду. Такие устройства называют интенсификаторами теплообмена [1-4]. Ясно, что если температура охлаждаемого объекта незначительно превышает температуру окружающей среды, такое применение оправданно, но, если температура объекта заметно выше температуры окружающей среды, непонятно, какие преимущества по сравнению с пассивным охлаждением имеет термоэлектрический интенсификатор теплообмена. В данном исследовании проводится анализ работы такого интенсификатора и даются критерии эффективности его работы.

\section{2. Рассмотрение работы интенсификатора}

Рассмотрим два способа охлаждения нагретого объекта, выделяющего тепло мощностью $Q_{0}$. Первый способ (рис. $1, a)$ предполагает, что тепло от объекта 1 сбрасывается на теплообменник с окружающей средой, который будем условно называть радиатором 2.

Во втором случае между радиатором и объектом помещается охлаждающий термоэлектрический модуль 3 (рис. $1, b)$. Попытаемся выяснить, позволяет ли использование термоэлектрического охладителя более эффективно понижать температуру охлаждаемого объекта, если эта температура даже после использования термоэлектрического охладителя остается выше температуры окружающей среды $T_{a}$, т. е. $T_{0}>T_{a}$.

Для случая, представленного на рис. $1, a$, связь между перепадом температур между объектом и окружающей средой, $\Delta T_{r}$, дается выражением

$$
\Delta T_{r}=T_{2}-T_{a}=R_{r} Q_{0},
$$

где $R_{r}$ - тепловое сопротивление радиатора 2 .

При использовании дополнительного термоэлектрического охладителя уравнение теплового баланса на холодном и на горячем концах модуля имеет вид

$$
\begin{aligned}
& \alpha I T_{0}-\frac{1}{2} I^{2} R-K \Delta T_{1}=Q_{0}, \\
& \alpha I T_{1}+\frac{1}{2} I^{2} R-K \Delta T_{1}=Q_{1},
\end{aligned}
$$

где $\alpha$ - суммарная термоэдс ветвей модуля, $I-$ ток через модуль, $R$ - электрическое сопротивление модуля, $K$ - теплопроводность модуля, $\Delta T_{1}=T_{1}-T_{0}$ разность температур на модуле, $Q_{1}$ - тепло, выделяющееся на горячем конце модуля. Температурные зависимости величин $\alpha, R, K$ не учитываются.

В случае, представленном на рис. $1, b$, перепад температур на радиаторе охлаждения равен

$$
\Delta T_{m r}=T_{1}-T_{a}=R_{r} Q_{1}=R_{r} \mu Q_{0},
$$

где $\mu=Q_{1} / Q_{0}$ - тепловой коэффициент.

$a$
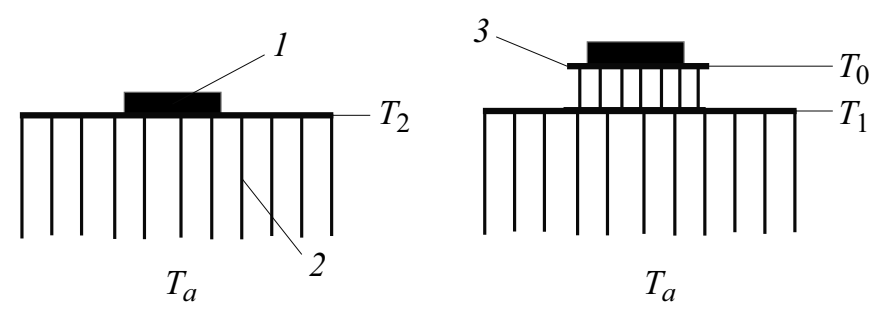

Рис. 1. Система охлаждения объекта 1 с помощью радиатоpa 2 (a) и система охлаждения того же объекта с помощью термоэлектрического модуля 3 и того же радиатора $(b)$. 
Для того чтобы схема с термоэлектрическим охлаждением работала эффективнее простой схемы с радиатором, требуется температура на объекте при использовании термоэлектрического охладителя (см. рис. $1, b)$ меньше, чем температура в случае рис. $1, a$, т. е.

$$
\Delta T_{r}>\Delta T_{m r}-\Delta T_{1}=\mu \Delta T_{r}-\Delta T_{1},
$$

где $\mu$ - тепловой коэффициент. Учитывая связь между холодильным и тепловым коэффициентом $\varepsilon=\frac{1}{\mu-1}$, получаем

$$
\Delta T_{1} \varepsilon \geq \Delta T_{r} .
$$

При выполнении неравенства (5) использование термоэлектрического охладителя позволяет снизить температуру объекта по сравнению с охлаждением только радиатором. Поэтому (5) является основным критерием для оценки эффективности работы термоэлектрического интенсификатора теплообмена. Из (5) также видно, что увеличение $\varepsilon$ позволяет увеличивать допустимую величину $\Delta T_{r}$.

Рассмотрим случай работы модуля в режиме максимальной энергетической эффективности (максимального $\varepsilon$ ), что позволяет минимизировать тепло, выделяющееся на горячей стороне модуля. Для этого режима известны выражения [3]

$$
I=\frac{\alpha \Delta T_{1}}{R(M-1)}
$$

где

$$
\begin{gathered}
M=\sqrt{1+Z \frac{T_{0}+T_{1}}{2},} \\
Z=\frac{\alpha^{2}}{R K} .
\end{gathered}
$$

В таком режиме максимальная холодопроизводительность достигается при $\Delta T_{1} \approx \Delta T_{\max } / 2$ и $\varepsilon \approx 1$, где $\Delta T_{\max }$ - максимальная разность температур на модуле. Из (5) получается приблизительная оценка для $\Delta T_{r} \leq T_{\max } / 2$, при которой использование дополнительного термоэлектрического модуля оправданно. Более детальное рассмотрение проблемы дается далее.

Соотношения между температурами (определение ясно из рис. 1) можно записать в виде

$$
\begin{gathered}
T_{1}=T_{a}+\mu \Delta T_{r}, \\
T_{0}=T_{a}+\mu \Delta T_{r}-\Delta T_{1},
\end{gathered}
$$

где $\mu-$ тепловой коэффициент, равный $\mu=1+1 / \varepsilon$. Из выражения (5) следует, что существует максимальное значение $\Delta T_{r}=\Delta T_{r \max }$, при превышении которого ввод термоэлектрического модуля в систему теплосброса охлаждаемого объекта приводит не к улучшению, а к ухудшению условий теплообмена. Это условие соответствует равенству температур

$$
T_{2}=T_{0},
$$

что дает в соответствии с (5) равенство $\Delta T_{1}=\Delta T_{r} / \varepsilon$. Из выражения для холодильного коэффициента при работе в режиме максимальной энергетической эффективности

$$
\varepsilon=\frac{M T_{0}-T_{1}}{\Delta T_{1}(M+1)}
$$

с учетом (9)-(11) можно получить

$$
M_{\varepsilon}=\frac{T_{a} \varepsilon+(2 \varepsilon+1) \Delta T_{r}}{T_{a} \varepsilon},
$$

где

$$
M_{\varepsilon}=\sqrt{1+Z T_{a}+Z \Delta T_{r}\left(1+\frac{1}{2 \varepsilon}\right)} .
$$

Учитывая для реальных величин $Z$ справедливость оценки $Z \Delta T_{r}(1+1 / 2 \varepsilon) \ll 1+Z T_{a}$, для $M_{\varepsilon}$ можно принять приближенное выражение

$$
M_{\varepsilon} \approx M_{a}\left(1+\frac{Z \Delta T_{r}(2 \varepsilon+1)}{4 M_{a}^{2} \varepsilon}\right),
$$

где

$$
M_{a}=\sqrt{1+Z T_{a}} .
$$

Подставляя (15) в (13), можно получить оценку

$$
\Delta T_{r \max }=\frac{\varepsilon T_{a}\left(M_{a}-1\right)}{(2 \varepsilon+1)\left[1-\frac{Z T_{a}}{4 M_{a}}\right]},
$$

где вторая дробь - медленно меняющаяся с $Z$ функция, поэтому для небольших изменений $Z$ величина $\Delta T_{r \max }$ линейно зависит от $Z$.

На рис. 2 для примера приведена зависимость $\Delta T_{r \text { max }}$ от $Z$ при различных значениях холодильного коэффициента и $T_{a}=300 \mathrm{~K}$.

Из рис. 2 видно, что при малых $\varepsilon$ предельная разность температур на радиаторе мала $(28-35 \mathrm{~K}$ для $\varepsilon=0.5)$. По мере возрастания $\varepsilon$ величина $\Delta T_{r \max }$ возрастает, достигая значений $57-64 \mathrm{~K}$ для $\varepsilon=10$.

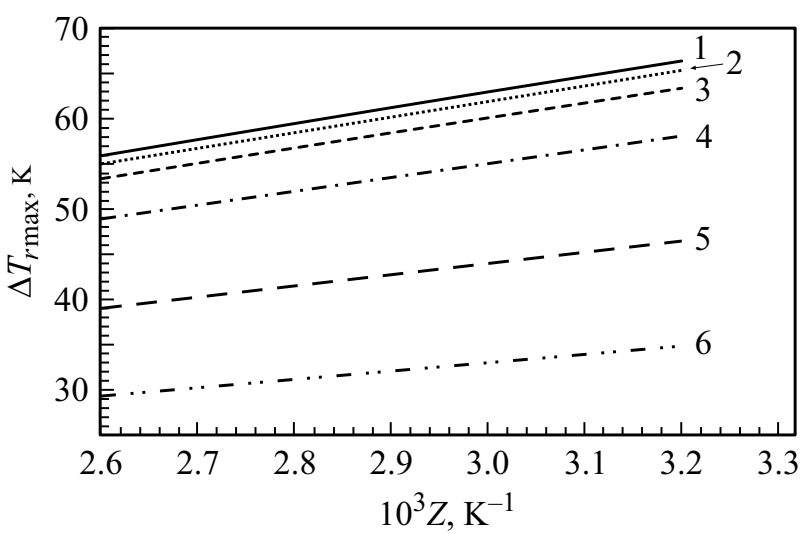

Рис. 2. Зависимость $\Delta T_{r \max }$ от $Z$ при $T_{a}=300 \mathrm{~K}$ для различных значений холодильного коэффициента $\varepsilon: 1-10,2-7.5$, $3-5,4-2.5,5-1,6-0.5$. 
Предельные значения $\Delta T_{r \max }$, при превышении которых использование термоэлектрических охладителей в цепи охлаждения объекта приводит к повышению его температуры при $T_{a}=300 \mathrm{~K}$

\begin{tabular}{c|c|c|c|c}
\hline$Z, 1 / \mathrm{K}$ & 0.0026 & 0.0028 & 0.003 & 0.0032 \\
\hline$T_{r \max }, \mathrm{K}$ & 58.7 & 62.4 & 66.1 & 69.7
\end{tabular}

При $\varepsilon \rightarrow \infty$, что соответствует $\Delta T_{1} \rightarrow 0$ (9), из можно получить предельные значения $\Delta T_{r \text { max: }}$ :

$$
\Delta T_{r} \rightarrow \frac{T_{a}\left(M_{a}-1\right)}{2\left(1-Z T_{a} / 4 M_{a}\right)},
$$

для которых использование термоэлектрического модуля, независимо от режима работы, только ухудшает ситуацию с теплообменом. Эти результаты приведены в таблице.

Полученные ограничения на температуру радиатора, приведенные на рис. 2 и в таблице, показывают, что для больших разностей температур на радиаторе введение термоэлектрического охладителя не приводит к снижению температуры объекта, что значительно сужает область применения термоэлектрических интенсификаторов теплообмена.

Использование термоэлектрических интенсификаторов теплообмена должно приводить к снижению температуры объекта на величину

$$
\Delta=T_{2}-T_{0}=\Delta T_{1}-\frac{\Delta T_{r}}{\varepsilon} .
$$

Из (7) следует, что

$$
M=\sqrt{1+Z T_{1}-Z \frac{\Delta T_{1}}{2}} \approx M_{1}-Z \frac{\Delta T_{1}}{4 M_{1}^{2}},
$$

где

$$
M_{1}=\sqrt{1+Z T_{1}}
$$

Из (12) с использованием (20) можно получить

$$
\begin{gathered}
x^{2} Z T_{1}(\varepsilon+1)-x\left[Z T_{1}+4 M_{1}^{2}(\varepsilon+1)+4 M_{1} \varepsilon\right] \\
+4 M_{1}\left(M_{1}-1\right)=0,
\end{gathered}
$$

где

$$
x=\frac{\Delta T_{1}}{T_{1}} .
$$

Решив (22) с учетом определения (9), можно получить соотношение между $\Delta T_{1}$ и $T_{1}$ для различных значений $Z$.

На рис. 3 приведены зависимости снижения температуры радиатора $\Delta$ в зависимости от перепада температур на радиаторе для двух разных величин и для наиболее близкой к встречающейся на реальных термоэлектрических модулях величины $Z=0.00281 / \mathrm{K}$. Из рисунка видно, что для больших значений эффект снижения температуры за счет использования термоэлектрического

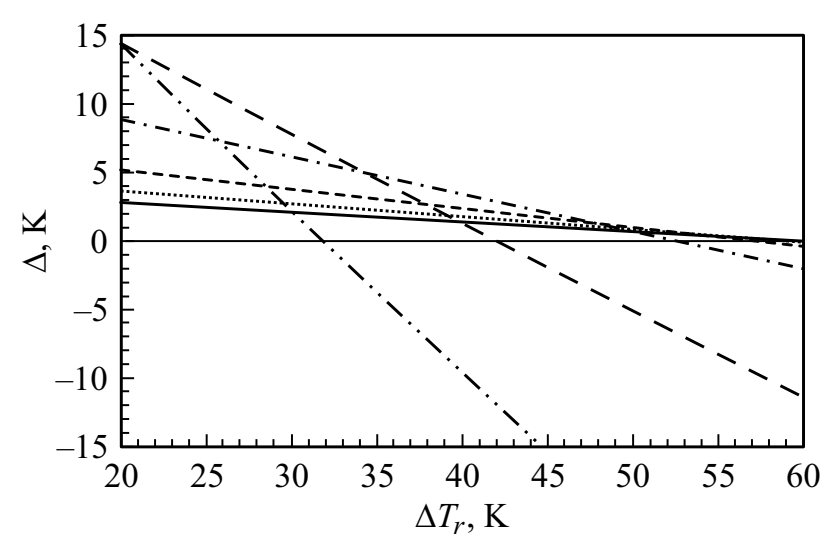

Рис. 3. Снижение температуры радиатора $\Delta$ в зависимости от перепада температур на радиаторе $\Delta T_{r}$ и холодильного коэффициента $\varepsilon$ при $T_{a}=300 \mathrm{~K}$ и $Z=0.00281 / \mathrm{K}$. Отрицательные значения $\Delta$ означают, что использование термоэлектрического модуля приводит к повышению температуры объекта. Обозначения те же, что на рис. 2.

интенсификатора теплообмена весьма незначителен (не превышает $5 \mathrm{~K}$ ). Наибольший эффект от применения интенсификатора $(\Delta=12-18 \mathrm{~K})$ наблюдается для малых $\varepsilon$ $(\varepsilon=1-2.5)$, но в той области, где температура объекта невелика $\left(\Delta T_{r}=20-30 \mathrm{~K}\right)$.

\section{3. Заключение}

Приведенное рассмотрение показывает, что для снижения температуры охлаждаемого объекта при больших значениях $\Delta T_{r}(>40 \mathrm{~K})$ необходимо, чтобы модуль работал в режиме больших $\varepsilon(\varepsilon=5-10)$, однако эффект снижения температуры при этом очень низок, и $\Delta$ в зависимости от величины $Z$ не превышает $5 \mathrm{~K}$. Таким образом, использование интенсификатора теплообмена в режиме больших $\varepsilon$ оказывается малоэффективным. Кроме того, переход к большим $\varepsilon$ сопровождается как уменьшением величины $\Delta T_{1}$, так и уменьшением тока через модуль (6). Это должно сопровождаться уменьшением холодопроизводительности модуля.

В качестве основной энергетической характеристики модуля обычно рассматривается холодопроизводительность при нулевой разности температур на модуле $Q_{\max }$ :

$$
Q_{\max }=\frac{K Z\left(T_{1}-\Delta T_{\max }\right)^{2}}{2}
$$

Для малых $\Delta T_{1}$, пренебрегая членами 2-го порядка по $\Delta T_{1} / T_{0}$, можно получить из (2) для холодопроизводительности в режиме максимальной энергетической эффективности

$$
Q_{0}=K \Delta T_{1}\left(\frac{Z T_{0}}{M-1}-1\right)
$$


Находя $K$ из (25) и подставляя в (24), получаем

$$
Q_{\max }=Q_{0} \frac{T_{0}}{\Delta T_{1}} \frac{Z T_{0}}{2\left(\frac{Z T_{0}}{M-1}-1\right)} \frac{\left.T_{1}-\Delta T_{\max }\right)^{2}}{T_{0}^{2}} .
$$

Из (26) следует, что для малых $\Delta T_{1} / T_{0}$ максимальная холодопроизводительность используемого модуля $Q_{\max }$ растет пропорционально $T_{0} / T_{1}$, а это увеличивает как размеры (при неизменной высоте ветви), так и стоимость модуля. Поэтому работать с малыми перепадами температур на термоэлектрическом интенсификаторе теплообмена, являющимися следствием больших $\varepsilon$, бесперспективно, как вследствие их малого теплового эффекта, так и по экономическим показателям.

Работа термоэлектрического модуля в области малых $\varepsilon<1$ представляется для улучшения теплообмена также малоперспективной. Такие режимы улучшают теплообмен только для разности температур на радиаторе $\Delta T_{r}<30-35 \mathrm{~K}$, при этом для снижения температуры объекта на $10 \mathrm{~K}$ величина $\Delta T_{r}$ не должна превышать $22-28 \mathrm{~K}$.

Таким образом, во многих случаях термоэлектрический охладитель интенсифицировать теплообмен не может. Небольшие перспективы имеют режимы с $\varepsilon=1-2.5$, которые могут обеспечить при $\Delta T_{r}=35-40 \mathrm{~K}$ снижение температур на $5 \mathrm{~K}$.

Для снижения температуры объекта необходимо одновременно с использованием термоэлектрического модуля уменьшать тепловое сопротивление радиатора. Однако если происходит замена радиатора, очевидно, что его можно подобрать так, чтобы исключить применение термоэлектрических модулей для дополнительного охлаждения. Это особенно касается случаев применения интенсификаторов в радиоэлектронной аппаратуре [5], где максимальная рабочая температура кремниевых активных элементов ограничена $80-90^{\circ} \mathrm{C}$. Для таких температур реальные значения $Z$ промышленно выпускаемых модулей находятся в пределах $(2.6-2.8) \cdot 10^{-3} \mathrm{~K}^{-1}[6]$, что делает, в соответствии с таблицей, бесперспективным использование термоэлектрических интенсификаторов теплообмена.

Представление о том, что при неэффективно работающем радиаторе термоэлектрический интенсификатор теплообмена эффективен, вытекает из метода расчетов таких интенсификаторов, при которых всегда одновременно с использованием термоэлектрического охладителя оптимизировался и радиатор. Для такой пары всегда можно подобрать оптимальную компоновку, но вопрос о том, нужен ли при этом вообще термоэлектрический охладитель, никогда не рассматривался.

\section{Конфликт интересов}

Авторы заявляют, что у них нет конфликта интересов.

\section{Список литературы}

[1] В.С. Мартыновский, В.А. Наер. Холодильная техника, № 3, 4 (1961).

[2] Д.А. Тайц. Холодильная техника, № 5, 26 (1970).

[3] Э.М. Лукишкер, А.Л. Вайнер, М.Н. Сомкин, В.Ю. Водолагин. Термоэлектрические охладители (М., Радио и связь, 1983) c. 27.

[4] Э.М. Лукишкер, А.Л. Вайнер. Вопросы радиоэлектроники, сер. ТПТО, № 1, 86 (1978).

[5] Д.К. Кадирова. Вестн. Дагестан. гос. техн. ун-та. Техн. науки, 44 (2), 68 (2017).

[6] П. Шостаковский. Компоненты и технологии, № 12, 40 (2009).

Редактор Л.В. Шаронова

\section{Thermoelectric heat transfer intensifiers}

\section{I.A. Drabkin, L.B. Ershova}

RMT Ltd.,

115230 Moscow, Russia

Abstract The work of a thermoelectric heat transfer intensifier is analysed in this paper. Criteria for the efficiency of such intensifiers are given. The advantage of using a thermoelectric cooler is evaluated depending on the temperature difference on the heat sink without a thermoelectric module. It is shown that in many cases a thermoelectric cooler cannot intensify heat transfer. In the case when a thermoelectric cooler is used to reduce the object temperature, it is also necessary to reduce the heat sink thermal resistance. 